\title{
Unusual presentation of Scaphoid Tuberculosis
}

\author{
Gomathi.R.G, Rajagopalan.B,Dhanasekar.T \\ Department Of TB And Chest Medicine ,Sri Ramachandra Medical College ,Porur,Chennai, \\ Tamil Nadu ,India
} Abstract: The scaphoid is an uncommon site for tuberculosis infection. 79 years old male patient presented to
us with wrist pain and difficulty in using the wrist. On examination he had swelling over dorsal aspect. The
diagnosis of osteoarticular tuberculosis was suspected after radiological and biologically study then confirmed
after histological study.Antibacillary chemotherapy during 12 months promoted healing and good outcome.

\section{Introduction}

Tuberculosis [TB] usually has prolonged course and not identified before it becomes a full blown disease. TB scaphoid is very rare accounting for less than $1 \%$ of all musculo skeletal TB. Scaphoid has a precarious vascularity in which the proximal pole receives the blood supply from the distal pole through the waist of scaphoid which is the narrowest and weakest part of scaphoid; hence fracture is very commonly suspected and most commonly occurs in waist causing proximal pole prone for avascular necrosis and bone resorption. TB scaphoid has been commonly reported due to dog bite or IV canulation ${ }^{12}$ but not otherwise. The osteoarticular localization is rare ; affecting the hand and the wrist remains exceptional(2-4\%).The diagnosis is usually delayed and misdiagnosed, but when discovered at an early stage, a well followed medical treatment is uaually enough to provide full healing

\section{Case report}

79 years old male patient with no history of any injury, presented to us with wrist pain and difficulty in using the wrist. On examination he had swelling over dorsal aspect, anatomical snuffbox tenderness with range of movements restricted due to pain but no localized warmth or erythema. There was no neuro vascular deficit. We took an X-ray and found that there ismore than three fourths resorption of proximal pole of scaphoid. We investigated the patient, Chest xray showed normal study .He had elevated polymorphs $72 \%$ (normal $45-70 \%$ ) in total bloodcount,C - Reactive Protein(CRP) is $2.4 \mathrm{mg} / \mathrm{dl}$ (normal is $0-0.6$ ), fasting sugars (FBS) 130 (normal $70-110 \mathrm{mg} / \mathrm{dl}$ ), post prandial sugars (PPBS) $142 \mathrm{mg} / \mathrm{dl}$ (normal 80 - 140), erythrocyte sedimentation rate (ESR) $42 \mathrm{~mm} / \mathrm{hr}$ (normal 4 - 30), calcium $10.4 \mathrm{mg} / \mathrm{dl}$ (normal 8.5 - 10.1), globulin $3.9 \mathrm{~g} / \mathrm{dl}$ (normal 2 - 3.5). He had decreased lymphocytes 22.5\% (normal 25 - 40) and monocytes $1.4 \%$ (normal 2 - 10) in total count, $\mathrm{Hb} 12.6 \mathrm{gms} / \mathrm{dl}$ (normal 13 - 17), PCV 37.3 (normal 40- 50\%).

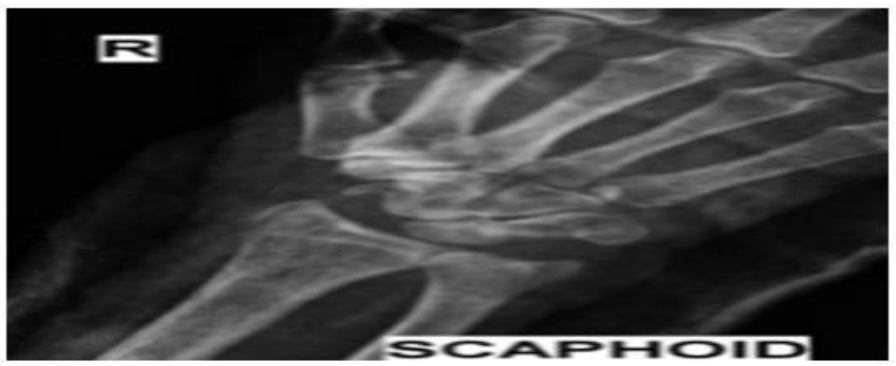

Figure 1 - Plain XR of wrist AP view showing more than three fourths resorption of scaphoid

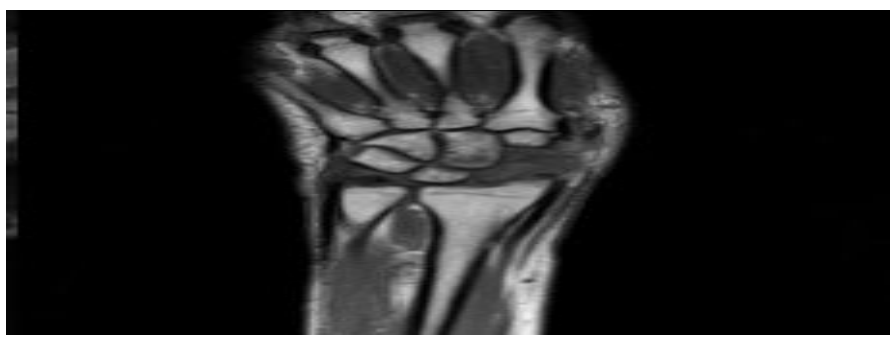

Figure 2 - MRI of wrist showed resorption of proximal three fourths of scaphoid with softtissue involvements. 


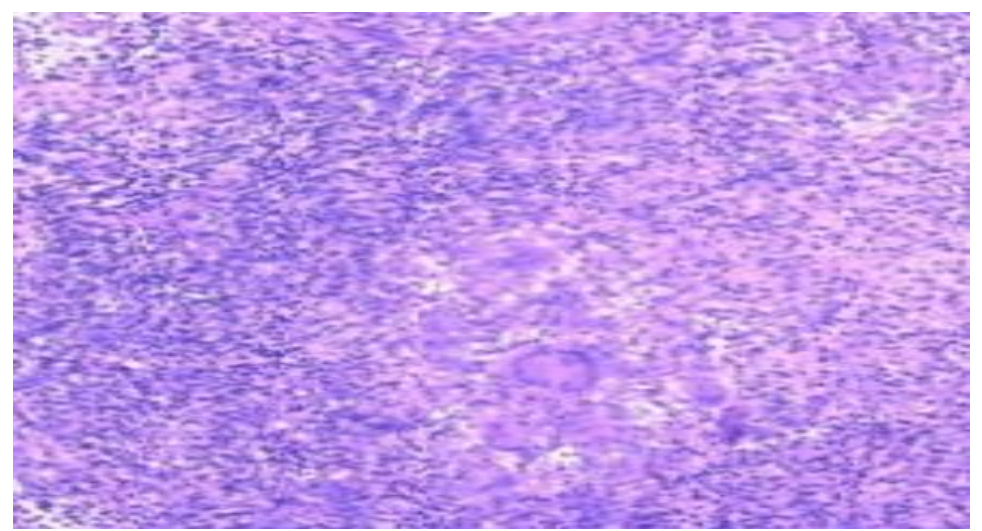

Figure 3 - Under higher resolution it showed slipper shaped epithelioid cells with Langhans Giant Cells surrounded by lymphocytes.

Magnetic resonance imaging (MRI) of the scaphoid bone showed resorption of proximal three fourths of scaphoid, except pisiform all other carpals are involved, small erosion in lateral aspect of distal radius, synovial thickening with effusion in lateral aspect of wrist, around scaphoid and along abductor pollicis longus and extensor pollicis brevis tendons. Nextdirect smear for AFB, Gram Staining, Fungal Stain and Tissue Culture all came as negative. ELISA screening for HIV for type 1 and 2 came negative.

biopsy of the scaphoid bone done and it came as tuberculous infection with granulomatous lesion.Under higher resolution it showed slipper shaped epithelioid cells with Langhans Giant Cells surrounded by lymphocytes.

Post operatively anti tuberculous treatment was started over a period of 12 months( associating isoniazid , rifampicin , pyrazinamide and ethambutol for the next 10 months ) promoted healing and a good health outcome in this patient: After two months there was excellent range of movements in the wrist with no pain.

\section{Discussion}

The incidence of TB has increased, even in the developed countries. Several risk factors can be considered responsible for the increased incidence of TB :the absence of BCG Vaccination ,trauma or immunodeficiency (which can reactivate a pre-existing tubercular infection )and low socioeconomic status (which seems to be the major factor ). TB of hands and wrist is the rarest osteoarticular localization after shoulder .It represents $2-4 \%$ of all the localizations of the musculoskeletal system . Chronic wrist pain is among the most difficult to diagnose ${ }^{3}$.The most common non traumatic causes of wrist pain are tendinitis and nerve problems. Causes of symptomatic inflammation of upper extremity tendons and peritendons are poorly understood $^{4}$.Tuberculosis of wrist is very rare ${ }^{5}$.Foci of infection is usually distal radius and primarily in synovium. From there it permeates into carpals and flexor and extensor tendons. In patients with normal immunity tuberculous infection starts as synovitis and the course is slow. There will besynovial effusion and granulation. The granulation tissue extends onto the bone through synovial reflections and erodes the bone 6 .

TB bacilli usually reach the bone through blood via sub synovial vessels directly or indirectly from lesions in epiphyseal bone eroding into joint space. Rarely infection can start in bone and cross infect synovium ${ }^{11}$. If abscess forms it penetrates the bone and periosteum and migrates in various directions along fascial planes. Bone destruction occurs by lysis ${ }^{8}$.ESR and CRP may be increased but they are also increased in many other conditions ${ }^{7}$ and not specific but can be sensitive to indicate occult disease ${ }^{9}$.

Mohammed Azfar Siddiqui et al in their paper stressed that identifying primary foci will always be difficult ${ }^{12}$.Clinical and radiological diagnosis will give scant information and only high degree of suspicion can lead to diagnosis.Our case had no foci of tuberculosis anywhere else. Nor immune suppressed. He had mild anemia with polymorphosis, newly diagnosed diabetes mellitus only during this hospital visit, not taking any medications, no history of radial artery or any form of IV cannulations in forearm or hand recently. With no predisposing factors he developed TB synovitis of abductor pollicis longus and extensor pollicis brevis, eroded the adjacent lateral aspect of distal radius. Biopsy reported focal areas of abscess which invaded and eroded the proximal scaphoid. He came to as two months after the symptoms started. On taking x-ray we found bony erosion of proximal scaphoid and for two months duration it looked malignant. But when evaluated fully it came as tuberculosis. 
Generally in patients with tuberculosis skeletal involvement occurs in 2- 3\%. Even among these patients involvement of small bones like carpals is rare. When a patient comes to us with pain and swelling in the wrist and clinically having anatomical snuffbox tenderness the first diagnosis will be scaphoid fracture. Even if there is no history of trauma, we can suspect osteoporotic fracture in elderly individuals like our case. Next to fracture, infection should be suspected even if there is no localized sign of infection.Pinar Borman et al in their article ${ }^{13}$ has suggested that recent trends show that chances of tuberculosis infection shift to older population with involvement of unusual sites.

Pinar Bormanet $\mathrm{al}^{13}$ has stated that in patients with tuberculous wrist infections constitutional symptoms are usually absent and mostly they present with pain and swelling. Our case had pain, swelling with restricted range of movements in the wrist but no constitutional symptoms.

Kotwal et $\mathrm{al}^{14}$ has described after reviewing 32 cases that the treatment can include anti tuberculous treatment for eight weeks. Only in those cases where there is no response we should do open or arthroscopic debridement. Incision and drainage of abscesses can be done.The treatment consists of anti tuberculous treatment with immobilization with slab or cast in functional position is the preferred treatment.

After the acute phase goes off wrist arthrodesis in $10^{0}$ can be considered ${ }^{2}, 10$.

chemotherapy : The classic long term treatment (1 year) has been replaced in recent years with a progressively shorter treatment (6 months )using more effective drugs

Our case has some typical features like $\mathrm{x}$ - ray showed only resorption of three fourths of scaphoid without showing involvement of any other structure. The duration is very short with no history of trauma and the amount of erosion with such a short duration in an old man made us suspect malignancy, on presentation he had pain, swelling with restricted movements but no other signs to suspect infection.

\section{Conclusion}

Skeletal involvement occurs in 2-3\% of patients suffering from tuberculosis .TB of the wrist begins in the scaphoid ,but is usually diagnosed at an advanced stage of carpal destruction, due to the slow development of the symptoms. At an early stage, the affection of the scaphoid must be highly suspected of being osteoarticular TB,especially in endemic areas. Antituberculosis treatment remains effective as a first choice with a good health outcome

\section{Bibliography}

[1]. Bettinger PC, Linscheid RL, Berger RA, Cooney WP, 3rd, An KN. An anatomic study of the stabilizing ligaments of the trapezium and trapeziometacarpal joint. J Hand Surg Am 1999;24-4:786-98.

[2]. Tuberculosis of bones \& joints,B. Sankaran. Ind Journal of Tuberculosis 1993, 40, 109.

[3]. Larry.K., Chronic wrist pain., Orthopaedic clinics of North America, Common Hand problems, Jan 1992, Page- 60

[4]. Eric Thorson MD., Robert M. Szabo. MD., Orthopaedic clinics of North America, Common Hand problems, Jan 1992, Page- 65

[5]. Tuberculosis by S. M. Tuli., $4^{\text {th }}$ edition page 158.

[6]. Tuberculosis by S. M. Tuli., $4^{\text {th }}$ edition page 11 .

[7]. Harrisons principles of Internal Medicine., 18thedition, page 875.

[8]. Tuberculosis by S. M. Tuli., $4^{\text {th }}$ edition page 13 .

[9]. Harrisons principles of Internal Medicine., 18thedition, page 145.

[10]. Hodgson 1972., Leung 1978, Tuli. S.M., Tuberculosis, $4^{\text {th }}$ edition page 161.

[11]. Tuli. S.M., Tuberculosis, $4^{\text {th }}$ edition page 10

[12]. Isolated tubercular scaphoid osteomyelitis:a case report, Mohammed Azfar Siddiqui, Orthopaedic Surgery (2012), Volume 4, No. 1, 64-66

[13]. Osteoarticular tuberculosis of the wrist,PinarBorman et al,Romatizma, Cilt: 17, SayÝ: 3, 2002

[14]. Tuberculosis of the hand,P. P. Kotwal et al., J Bone Joint Surg [Br] 2009;91-B:1054-7. 